\title{
Pore-scale velocities in three-dimensional porous materials with trapped immiscible fluid
}

\author{
Gaël Raymond Guédon ๑* and Fabio Inzoli ${ }^{\dagger}$ \\ Dipartimento di Energia, Politecnico di Milano, 20156 Milan, Italy \\ Monica Riva ${ }^{\ddagger}$ and Alberto Guadagnini ${ }^{\S}$ \\ Dipartimento di Ingegneria Civile e Ambientale, Politecnico di Milano, 20133 Milan, Italy \\ and Department of Hydrology and Atmospheric Sciences, University of Arizona, Tucson, Arizona 85721, USA
}

(Received 19 December 2018; revised manuscript received 31 May 2019; published 2 October 2019)

\begin{abstract}
We study and document the influence of wetting and nonwetting trapped immiscible fluid on the probability distribution of pore-scale velocities of the flowing fluid phase. We focus on drainage and imbibition processes within a three-dimensional microcomputed tomographic image of a real rock sample. The probability distribution of velocity magnitude displays a heavier tail for trapped nonwetting than wetting fluid. This behavior is a signature of marked changes in the distribution and strength of preferential flow paths promoted by the wettability property of the trapped fluid. When the latter is wetting the host solid matrix, high-velocity areas initially present during single-phase flow conditions are mainly characterized by increased or decreased velocity magnitudes, and the velocity field remains correlated with its counterpart associated with the single-phase case. Otherwise, when the trapped fluid is nonwetting, features that are observed to prevail are appearance and disappearance of high-velocity areas and a velocity field that is less correlated to the one obtained under single-phase conditions.
\end{abstract}

DOI: 10.1103/PhysRevE.100.043101

\section{INTRODUCTION}

Understanding and quantifying the impact of pore-scale flow heterogeneity on the observed behavior of fluid flow and transport at the continuum (Darcy) scale is key to the assessment of anomalous features documented in natural and engineered media under saturated $[1-8]$ and unsaturated conditions [9-14]. Knowledge of the probability distribution of pore-scale velocities has been demonstrated to be critical in determining dispersion mechanisms in porous media [15] and is a major element which allows advancing our ability to characterize fluid flow processes in porous media. Here we focus on the still unresolved challenging issue of characterizing the effects of a trapped immiscible fluid phase on the probability density distribution of pore-scale velocities of the flowing phase in the pore space of a natural rock.

Recent developments allowed characterizing the probability distribution of pore-scale velocities from the corresponding distribution of pore and throat sizes under single-phase flow conditions for two-dimensional porous microstructures $[16,17]$ and stochastically generated three-dimensional porous media [18]. These progresses have improved our understanding of the way low and high flow velocities distribute in a saturated porous medium characterized by well-defined pore size distribution and documented that a profound relationship exists between the underlying pore structure and fluid flow patterns. The situation is clearly more complicated in the

\footnotetext{
*Corresponding author: gaelraymond.guedon@polimi.it

†fabio.inzoli@polimi.it

${ }^{\ddagger}$ monica.riva@polimi.it

§alberto.guadagnini@polimi.it
}

presence of two or more fluid phases, and characterizing the probability distribution of pore-scale velocities represents a notable challenge from both an experimental and numerical point of view. Experiments are mostly limited by the spatial and temporal resolutions of measurement techniques and by the choice of flowing fluids and porous materials. Otherwise, high-resolution numerical methods applied to the simulation of multiphase flows mainly suffer from fictitious velocities originating from numerical artifacts at fluid-fluid interfaces and are associated with high computational costs. Okamoto et al. [19] analyze the influence of a stagnant immiscible liquid on the flow of water in porous media through a set of experiments performed in a three-dimensional bed of glass beads with and without the presence of trapped residual silicone oil. These authors use magnetic resonance imaging to characterize how localized high-velocity areas are modified by the presence of stagnant silicone oil and show that water velocity profiles can be drastically modified due to changes of (1) pore space accessible to fluid flow and (2) localization of high-velocity areas. Datta et al. [20] use confocal microscopy and refractive index matching to measure flow distribution in a three-dimensional bed of glass beads for single-phase flow and a flow condition characterized by trapped residual nonwetting oil. They show that the spatial distribution of pore-scale velocities is linked to the underlying pore structure and that the presence of nonwetting oil ganglia confines the flow to high-velocity, preferential paths. Jiménez-Martínez et al. $[11,14]$ analyze experimentally the dynamics of solute dispersion within two-dimensional porous media under unsaturated conditions and show that dispersion, mixing, and reaction rates are enhanced by increased flow confinement on preferential paths, due to the presence of trapped immiscible fluid phases. Despite these efforts, detailed descriptions of the 
TABLE I. Main characteristics of the porous rock sample analyzed.

\begin{tabular}{lcccc}
\hline \hline Number of voxels & $\begin{array}{c}\text { Sample size } \\
{\left[\mathrm{mm}^{3}\right]}\end{array}$ & Total porosity & Connected porosity & $\begin{array}{c}\text { Permeability }^{\mathrm{a}} \\
{\left[\mathrm{m}^{2}\right]}\end{array}$ \\
\hline $300 \times 300 \times 450$ & $1.2 \times 1.2 \times 1.8$ & $12.365 \%$ & $11.465 \%$ & $2.49 \times 10^{-13}$ \\
\hline \hline
\end{tabular}

${ }^{\mathrm{a} A s}$ determined from a single-phase flow simulation.

way pore-scale velocities distribute within natural pore spaces under multiphase conditions is still not completely explored. In this context, the effect of wettability of the trapped phase on flow confinement into preferential paths is still unexplored.

Here we rely on high-resolution numerical simulations to study and document the way low and high pore-scale flow velocities distribute in a three-dimensionally reconstructed Portland limestone for (1) single-phase flow and (2) multiphase flow with a trapped wetting or nonwetting immiscible fluid. These settings are representative of typical scenarios encountered in natural subsurface reservoirs and aquifers. We find that probability densities of low flow velocities follow a unique power-law behavior under both single- or multiphase conditions. Otherwise, high flow velocities display a stretched exponential behavior. The latter is characterized by a heavier tail for flow with a trapped nonwetting fluid phase, as compared to the remaining cases studied. We show that the wettability of the trapped phase plays a critical role in the way the residual phase distributes in the pore space and preferential paths are organized.

\section{MATERIALS AND METHODS}

We use typical simulators implemented in the OPENFOAM® toolbox [21], INTERFOAM and SIMPLEFOAM, to, first, numerically reproduce drainage and imbibition processes and, second, solve steady incompressible viscous flow of the flowing phase in the connected region resulting as a consequence of trapping phenomena. INTERFOAM uses a volume-of-fluid technique to implicitly track the interfaces between two immiscible flowing fluids on an Eulerian grid [22]. The relative amount of one of the fluid phases in a cell, i.e., the volume fraction $\alpha$, is solved in conjunction with the Navier-Stokes equations describing flow of the phases. Interfacial tension is accounted for through a balanced-force continuum surface force method [23,24] and calculating the interfacial curvature, $\kappa=-\nabla \cdot \mathbf{n}$, from the field of unit normal vector to the interface, $\mathbf{n}=\nabla \alpha /|\nabla \alpha|$. A one-fluid approach is used to formulate the system of conservation equations [25]. Mass conservation reads

$$
\frac{\partial \rho}{\partial t}+\nabla \cdot(\rho \mathbf{u})=0
$$

where $t$ is time and density $\rho=\alpha \rho_{1}+(1-\alpha) \rho_{2}$ is defined as a weighted sum of phase 1 (e.g., water) and phase 2 (e.g., oil) densities (subscripts 1 and 2 referring to phase 1 and 2 , respectively, and $\alpha=\alpha_{1}$ ). Velocity, $\mathbf{u}$, is rendered through the momentum balance equation

$$
\frac{\partial \rho \mathbf{u}}{\partial t}+\nabla \cdot(\rho \mathbf{u u})=-\nabla p+\nabla \cdot\left[\mu\left(\nabla \mathbf{u}+\nabla \mathbf{u}^{\top}\right)\right]+\sigma \kappa \nabla \alpha .
$$

Here $p$ denotes pressure, $\mu=\alpha \mu_{1}+(1-\alpha) \mu_{2}$ is dynamic viscosity, and $\sigma$ is the surface tension coefficient. Evaluation of transport of the volume fraction, $\alpha$, is typically plagued by numerical diffusion leading to smeared interfaces through time. In INTERFOAM, this smearing issue is mitigated by adding an artificial compression term to the volume fraction advection equation. This yields

$$
\frac{\partial \alpha}{\partial t}+\nabla \cdot(\alpha \mathbf{u})+\nabla \cdot\left[\alpha(1-\alpha) \mathbf{u}_{r}\right]=0 .
$$

Here $\mathbf{u}_{r}$ is a velocity field aligned with $\mathbf{n}$ and suitable to compress the interface, thus minimizing smearing [22].

We solve the set of governing equations within the pore space reconstructed from a three-dimensional microtomographic image of a Portland limestone (with a $4 \mu \mathrm{m}$ resolution) whose characteristics are listed in Table I. We extract pore network information (listed in Table II) upon relying on the marker-based watershed segmentation method of Gostick [26]. Applying traditional Dirichlet boundary conditions for pressure at the inlet and outlet boundaries of the simulation domain leads to strong capillary end effects affecting the two-phase flow solution [27]. We deal with this issue by (1) reflecting the pore space along the mean flow direction (Fig. 1) and (2) applying periodic boundary conditions in a way similar to Ramstad et al. [28]. To force flow in a given direction, we add a pressure drop between the inlet and outlet faces. A nearly constant flow rate is enforced by dynamically correcting the pressure drop. No-slip conditions are implemented at the remaining boundaries. Modeling contact line and contact angle dynamics is still a matter of debate and requires considering small-scale effects down to the molecular level [29]. To maintain a robust approach, we implement a model of constant contact angle at the fluid-solid interfaces. Numerical solution of the system of governing equations is achieved using a predictor-corrector technique [30] and a preconditioned conjugate gradient linear solver [31]. Stability of the simulation is ensured through convective and capillary time-step constraints. We do so by (1) limiting the largest travel distance a fluid particle can reach within a time step

TABLE II. Main characteristics of the pore network extracted from the rock sample analyzed following the marker-based segmentation method of Gostick [26]. Here $N_{p}$ and $N_{t}$ are the number of pores and throats, respectively; $\left\langle d_{p}\right\rangle$ is the average pore volumeequivalent diameter; $\left\langle d_{t}\right\rangle$ is the average area-equivalent throat diameter; and $\langle\mathrm{CN}\rangle$ is the average coordination number, representing a measure of the pore network connectivity.

\begin{tabular}{lcccc}
\hline \hline$N_{p}$ & $\left\langle d_{p}\right\rangle[\mu \mathrm{m}]$ & $N_{t}$ & $\left\langle d_{t}\right\rangle[\mu \mathrm{m}]$ & $\langle\mathrm{CN}\rangle$ \\
\hline 1227 & 54.1 & 1650 & 32.5 & 2.69 \\
\hline \hline
\end{tabular}




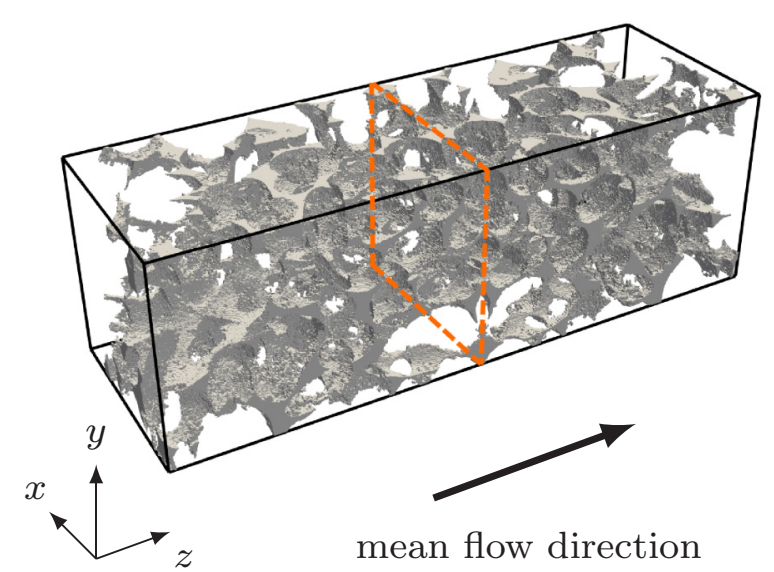

FIG. 1. Three-dimensional representation of the pore space showing the reflection of the porous structure along the mean flow direction; dashed lines denote the plane of reflection.

to a quarter of the grid spacing, $\Delta x$, and (2) guaranteeing that the propagation of capillary waves is temporally resolved. It is crucial to avoid aliasing of the shortest numerically represented capillary waves, whose physical motion cannot be accurately resolved [32]. Accordingly, the time-step size, $\Delta t$, should be driven by the constraint

$$
\Delta t \leqslant \min \left[0.25 \frac{\Delta x}{u_{\max }}, \sqrt{\frac{\left(\rho_{1}+\rho_{2}\right) \Delta x^{3}}{4 \pi \sigma}}\right] .
$$

Here $u_{\max }$ denotes maximum velocity magnitude, $\rho_{1}$ and $\rho_{2}$ are phase 1 and phase 2 fluid densities, respectively, and $\sigma$ is the surface tension coefficient. We simulate (primary) drainage by first saturating the porous sample with the wetting phase and then by injecting the nonwetting phase uniformly across the inlet. In our periodic setting, injection of the nonwetting phase takes place by keeping a constant unit volume fraction of the nonwetting phase in each of the void cells at the inlet plane. Likewise, (secondary) imbibition is simulated by maintaining a unit volume fraction of the wetting phase at the inlet, the initial fluid distribution being set as the final state of the drainage process. In both cases, simulation is run until steady-state conditions are attained, a scenario corresponding to approximately five injected pore volumes in our setting. To avoid misinterpretations of the probability distributions of pore-scale velocities, caused by spurious currents near the fluid-fluid interfaces, the connected region of the flowing fluid phase is extracted from the two-phase flow simulation and used as the computational domain for steady-state single-phase flow simulations. We perform these simulations using the SIMPLEFOAM solver. Here the solution of the steady incompressible viscous fluid flow is obtained through an iterative segregated method [33]. Considering stationarity of fluid-fluid interfaces at the final state of the two-phase flow simulations, we assume fixed computational domain boundaries, formed by an inlet, an outlet, and fluidsolid and fluid-fluid interfaces. In this setting, inlet and outlet faces of the pore space are made periodic, and a fixed pressure jump is set. No-slip boundary conditions are implemented for the fluid-solid interfaces. The boundary at the fluid-fluid
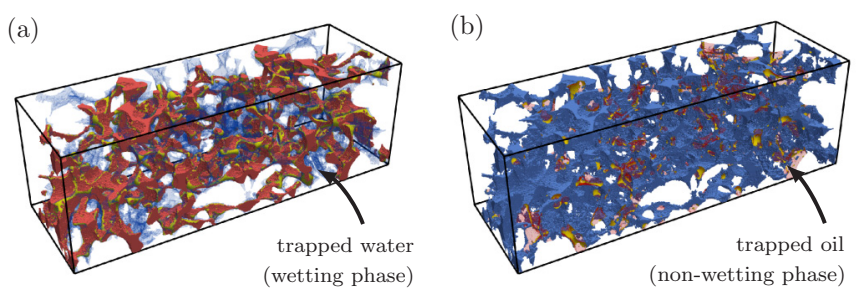

FIG. 2. The final fluid distribution obtained after primary drainage is shown in (a) where the invading oil is in red and the trapped residual water is represented in translucent blue; oil-water interfaces are in yellow. Likewise, the final state of the secondary imbibition is depicted in (b) with the invading water in blue and the trapped residual oil in translucent red.

interfaces is impermeable, with continuity of viscous stresses. This condition simplifies to a no-slip or to a free-slip condition, depending on the viscosity of the trapped phase being either infinite or zero. Similar velocity distributions are obtained in these two limit cases, as illustrated in Appendix A, suggesting that the influence of the fluid-fluid interface boundary condition is negligible for the scenarios investigated in this study. Results associated with the no-slip condition at the fluid-fluid interfaces are discussed in the following.

\section{RESULTS AND DISCUSSION}

\section{A. Simulation scenarios}

We start by evaluating the single- (wetting) phase steadystate flow in the available pore space as a baseline for comparison. We then simulate the flow of oil (with subscript $n w$, nonwetting phase) and water (with subscript $w$, wetting phase) characterized by a density ratio $\gamma=\rho_{n w} / \rho_{w}=0.78$ and by a viscosity ratio $\eta=\mu_{n w} / \mu_{w}=2.87$, values typically encountered in two-phase core-flooding experiments [34]. Water wets the solid with a uniform contact angle of $60^{\circ}$. The macroscopic pressure gradient along the $z$ axis is automatically adjusted during the simulation so that the operating capillary number is $\mathrm{Ca}=\mu_{n w} u_{D} / \sigma \approx 3 \times 10^{-5} \pm 10 \%$. Here $u_{D}$ is Darcy velocity calculated from the total volumetric flow rate. Accordingly, a flow regime slightly above capillarydominated conditions $\left(\mathrm{Ca}<10^{-6}\right)$ is achieved [35]. Stokes flow condition is verified and maintained in all simulations. Oil percolates in the pore space during primary drainage, and the total residual amount of (trapped) water after drainage occupies approximately $35.8 \%$ of the pore volume. Conversely, the residual (trapped) oil after imbibition accounts to approximately $34.6 \%$ of the pore volume. A three-dimensional rendering of the final fluid distribution is given in Fig. 2(a) and in Fig. 2(b) for the drainage and imbibition scenarios, respectively. We finally simulate steady single-phase flow in the connected region attained after primary drainage or secondary imbibition. Our analysis is therefore based on three settings: (1) single-phase flow of the wetting phase, (2) flow of the nonwetting phase with trapped wetting phase (trapped water), and (3) flow of the wetting phase with trapped nonwetting phase (trapped oil). We perform our computations using 3264 cores from the Marconi A2 partition of the Italian high-performance computing center, CINECA [36]. The total 

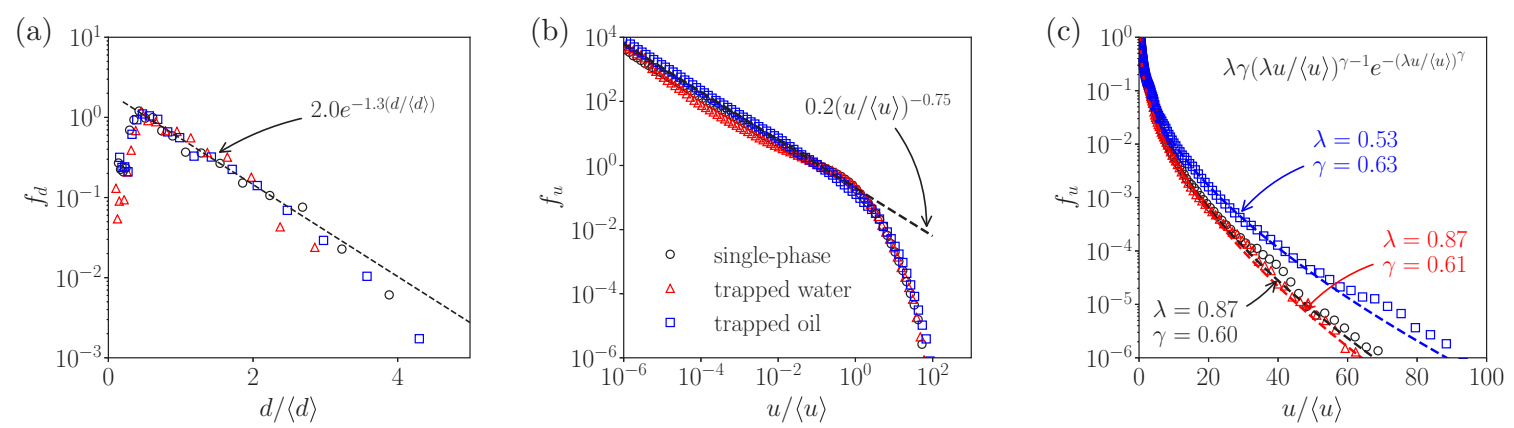

FIG. 3. (a) Probability density functions, $f_{d}$, of scaled pore volume-equivalent diameter, $d /\langle d\rangle$, and (b) probability density functions, $f_{u}$, of scaled velocity magnitude, $u /\langle u\rangle$, for the three settings analyzed. Symbols correspond to the simulation results and the straight dashed line to the power-law model employed to interpret the low-velocity range. (c) Semilogarithmic plot of the same data as in (b) with the stretched exponential functions considered to interpret the pattern of the decays observed in the distribution of high velocities.

computation time required for the study amounts to about 1500000 core hours, equivalent to 20 days.

\section{B. Probability distributions of pore-scale velocities}

The probability distribution of scaled velocity magnitudes, $f_{u}$ [Fig. 3(b)] displays a power-law scaling in the range of low velocities, $f_{u} \sim 0.2 \times(u /\langle u\rangle)^{-0.75}$, independent of the scenario considered, $\langle u\rangle$ being the mean of the (spatially variable) values of $u$ corresponding to a given scenario. An appraisal of the influence of the sample size on these types of results is included in Appendix B. Power-law scaling of low-velocity probability distributions was also observed in de Anna et al. [16] for single-phase flow in five two-dimensional geometries of nonoverlapping circular posts of random position and radius. These authors show that in their setting the exponent of the power law is directly proportional to the exponent of the power-law scaling characterizing the low range of the pore throat size probability distribution. Here we assess the probability distribution of pore and throat sizes of the medium and of the pore spaces formed after trapping using the marker-based watershed segmentation method of Gostick [26]. A close relationship between the probability distributions of low pore-scale velocities and throat sizes is not evident. Otherwise, considering the pore volume-equivalent diameter, $d$, as a metric for comparison, we find that the probability distribution of scaled $d, f_{d}$ [Fig. 3(a)], remains similar between the settings analyzed and is described by an exponential model, $f_{d} \sim 2.0 e^{-1.3(d /\langle d\rangle)}$, for $d /\langle d\rangle>0.5,\langle d\rangle$ being the mean of $d$. The similarity observed for the probability distribution of low pore-scale velocities in all settings analyzed is thus supported by the probability distribution of pore volume-equivalent diameters.

The high-velocity range of $f_{u}$ [Fig. 3(c)] displays a tail that is captured by a stretched exponential model (see Appendix B for an assessment of the influence of the sample size on the tail of $f_{u}$ )

$$
f_{u} \sim \lambda \gamma(\lambda u /\langle u\rangle)^{\gamma-1} e^{-(\lambda u /\langle u\rangle)^{\gamma}} .
$$

Stretched exponential tails in the probability distribution of pore-scale velocities were previously encountered in Siena et al. [18] for single-phase flow in nine stochastically generated three-dimensional porous media and in Siena et al.
[37] for single-phase flow in two natural porous media. Interpretation of the tail through the stretched exponential model is achieved for $u /\langle u\rangle>10$. The value of the stretching exponent, $\gamma$, does not change significantly across the cases considered. Otherwise, the scale factor, $\lambda$, displays a lower value (of about $40 \%$ ) in the presence of a trapped nonwetting phase as compared to the other two cases, which are characterized by the same estimated value of $\lambda$. This suggests that trapping of a nonwetting phase can give rise to heavier tailing.

\section{Role of wettability on main features of velocity distributions}

The results illustrated in Sec. III B document that the presence of a trapped phase can alter the probability distribution of high pore-scale velocities, shifting it toward higher values. This modification is even more pronounced when the trapped phase is nonwetting, revealing that preferential flow paths may undergo significant changes, depending on the wettability of the trapped fluid phase. We compare qualitatively the scaled velocity fields across the settings analyzed. Figure 4 depicts contours of scaled velocity on two planes perpendicular to the mean flow direction. We note that the spatial distribution of regions where (wetting and/or nonwetting) phase trapping can be observed is mainly governed by the pore-space geometry and by the contact angle formed at the triple fluid-fluid-solid contact lines. The effect of additional parameters (including the capillary number) can lead to spatial distributions of the trapped phase differing from what one could assess solely on the basis of geometrical attributes of the pore space. Under flow condition with trapped wetting (water) phase, we observe that water resides in the dead-end and high-entry pressure pores (i.e., pores that oil could not invade) as well as in the small crevices and corners of the pore space. Otherwise, when the trapped phase is nonwetting (oil), isolated oil ganglia are formed and occupy the central body of some pores and throats. Such a behavior is expected from theoretical arguments and was previously observed in several experiments [35]. In terms of velocity field, four significant features can be observed: high-velocity areas initially present in the single-phase case may be subject to (A) an increase or (B) a decrease in velocity magnitude, eventually until (C) velocity vanishes; a highvelocity area may also (D) appear in the pore space. When the trapped phase is wetting (water), we note that features 

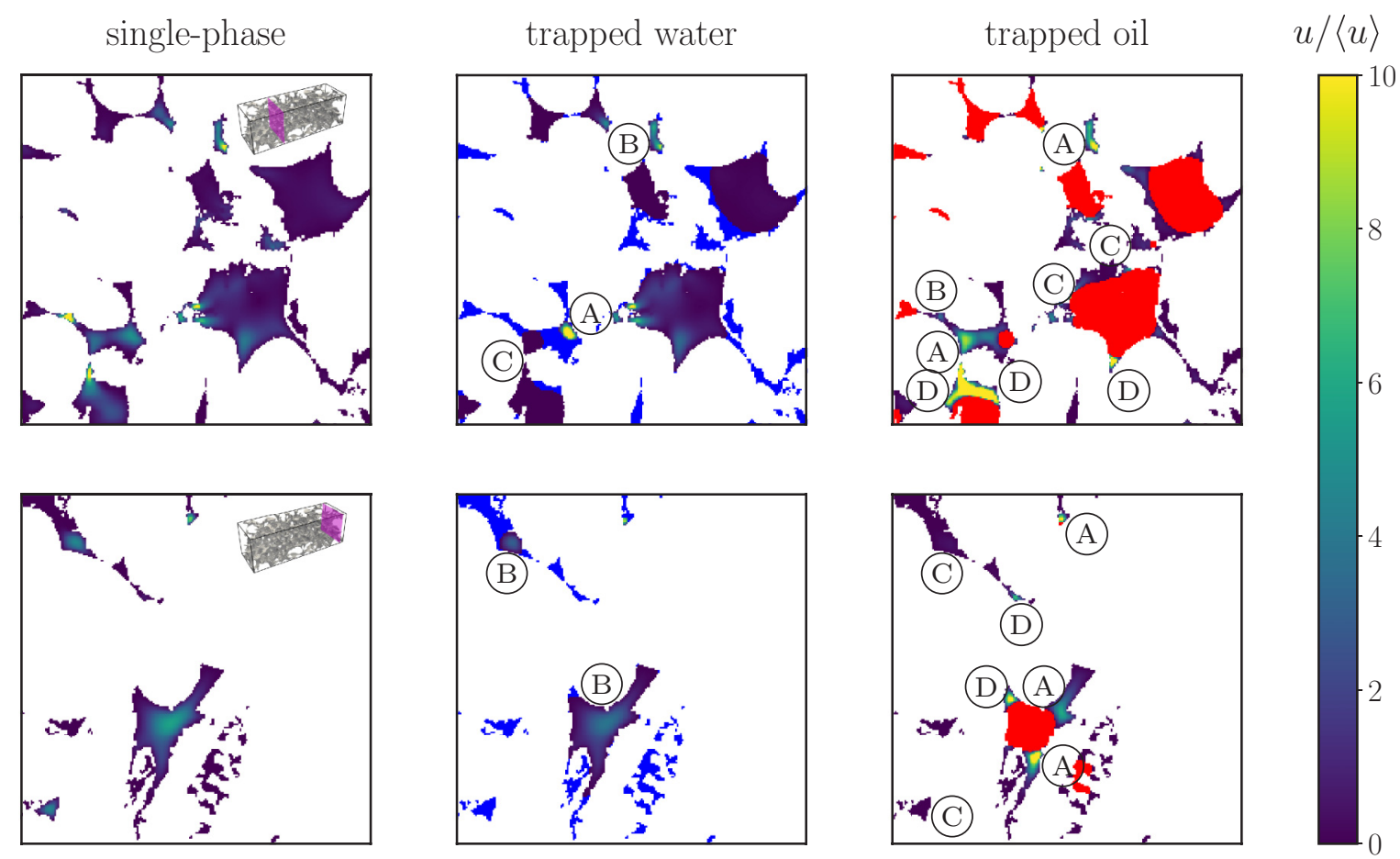

FIG. 4. Filled contours of scaled velocity magnitude on two planes normal to the mean flow direction for each case analyzed (top row: $z=0.34 L_{z}$; bottom row: $z=0.97 L_{z} ; L_{z}$ being the total length of the computational domain along the mean flow direction). Blue represents trapped water in the setting where oil is flowing (second column). Red represents trapped oil in the setting where water is flowing (third column). Symbols A and B, respectively, indicate higher and lower local flow velocities, as compared to the single-phase case. Symbols C indicate areas where velocity vanishes following trapping and D corresponding to appearance of high-velocity areas.

$\mathrm{A}, \mathrm{B}$, and $\mathrm{C}$ can be identified. In the second cross section analyzed (bottom row, second column in Fig. 4), only feature $\mathrm{B}$ is observed. Otherwise, all four features are documented when the trapped phase is nonwetting. To further support these observations, we compute scatter-plots of the scaled velocities for each cell of the pore space that is in common between pairs of differing settings considered. Figure 5 depicts (1) the scatter plot juxtaposing scaled velocities corresponding to single-phase flow and fluid flow in the presence of trapped phase at a given cross section together with (2) the associated contour plots of scaled velocities. Corresponding scatter plots associated with the overall sample are depicted in Fig. 6. Points close to the parity line (in red) correspond to regions where features A or B occur. Otherwise, points close to the horizontal or vertical axis corresponds to locations associated with features C and D, respectively. Details on the way these features are identified in the scatter plot are provided in Appendix C. Comparing the solution obtained in the presence of a trapped wetting (water) phase against the single-phase solution on the whole sample [Fig. 6(a)], we note that feature D, i.e., appearance of high-velocity areas, is absent (clusters of points near the vertical axis are missing in the graph). Instead, signatures of high-velocity channels with either increased (feature A) or decreased (feature B) velocity magnitudes are mostly noted, as documented by the observation that points are mostly concentrated near the parity line. Comparing the solution obtained in the presence of a trapped nonwetting (oil) phase against the single-phase solution [Fig. 6(b)] evidences the occurrence of a relatively dense cloud of points near the vertical axis. This finding suggests that several areas that were characterized by low velocities (stagnant) under singlephase flow conditions are now associated with high velocities (feature D). Dense clouds of points near the horizontal axis (feature C) are also observed, with an ensuing decrease of points close to the parity line (features A and B). Similar observations can be made comparing the setting with trapped oil against the one with trapped water [Fig. 6(c)], suggesting that the latter is more correlated with the single-phase case than with the solution obtained following oil trapping. These results reveal that features A and B are promoted when the trapped phase is wetting and the distribution of preferential pathways is expected to be similar to the single-phase conditions. Otherwise, features $\mathrm{C}$ and $\mathrm{D}$ are mostly promoted when the trapped phase is nonwetting, with a more pronounced impact on the distribution of preferential pathways. As such, wettability is a significant parameter to take into account together with the degree of saturation when characterizing flow in porous media under multiphase conditions.

\section{CONCLUSIONS}

Our results lead to the following major conclusions.

(1) The probability density of pore-scale velocity magnitudes can be described by a power law in the low-velocity range, regardless of the presence of a trapped fluid phase. Probability densities in the range of high velocities follow a stretched exponential decay with a stretching exponent that is markedly lower in the presence of a trapped nonwetting phase. We show that this characteristic is linked to the spatial arrangement of the trapped fluid phase, that is in turn associated 
(a)

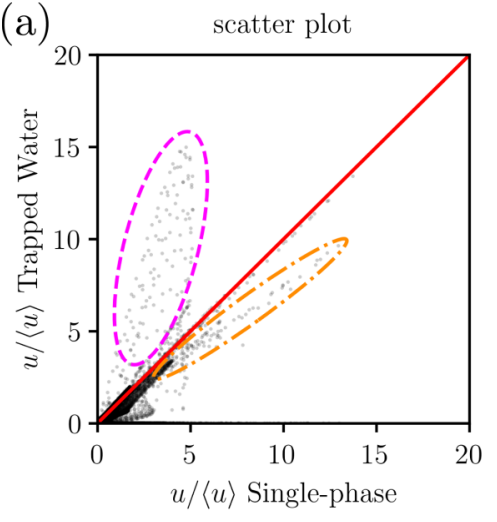

(b)

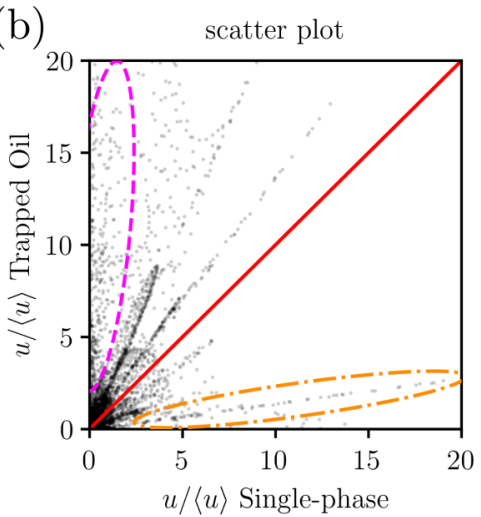

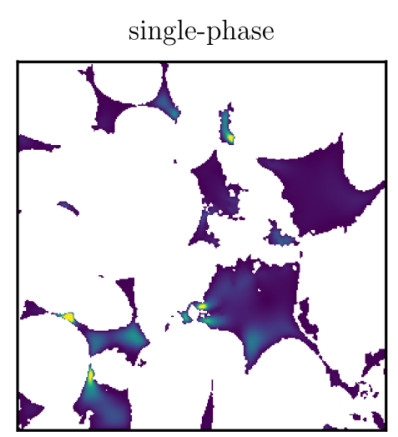
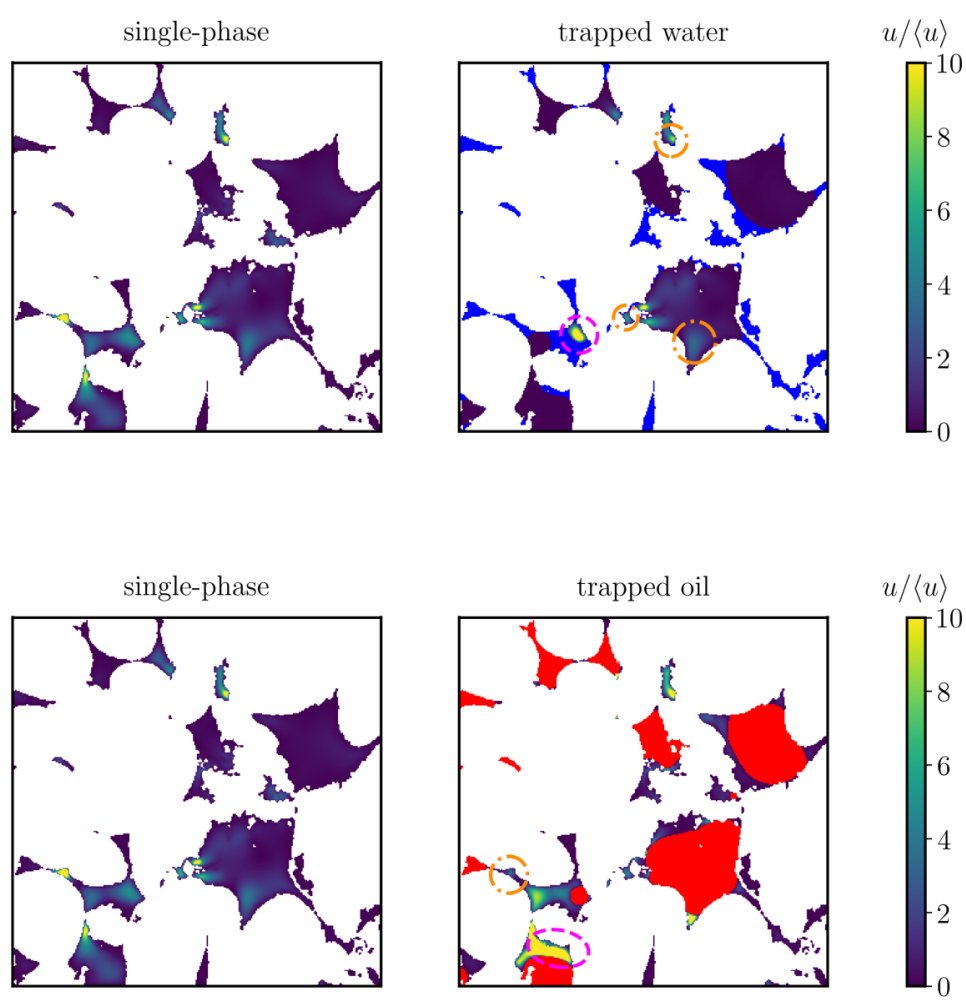

FIG. 5. Scatter-plot of scaled velocities corresponding to single-phase flow and fluid flow in the presence of trapped (a) water or (b) oil, for the cross section at $z=0.34 L_{z}$ (see Fig. 4). Associated contour plots are depicted for (middle column) single-phase flow condition and (right column) settings related to the presence of a trapped phase. Points close to the parity line (in red) correspond to regions where feature A or B occurs. Otherwise, points close to the horizontal or vertical axis correspond to locations associated with features C and D, respectively. These two sets of points and the corresponding spatial pattern are respectively identified through dashed magenta and dash-dotted orange curves (see Appendix $\mathrm{C}$ for additional details).

with the wettability of the trapped fluid. A trapped wetting phase distributes in the dead-end and high-entry pressure pores as well as in the small crevices and corners of the pore space and does not obstruct the central body of pores and throats, where the nonwetting phase flows. Otherwise, a trapped nonwetting phase resides in the form of isolated ganglia that remain in the central body of pores and throats and modifies the distribution of preferential pathways.
(2) Comparison of the velocity fields amongst the three settings analyzed (i.e., single-phase flow or two-phase flow in the presence of wetting or nonwetting trapped phase) leads to identifying four key features. High velocities associated with a single-phase flow condition can either increase or decrease, eventually approaching zero. High velocities can also appear within regions classified as stagnant (or nearly stagnant) under single-phase flow conditions.
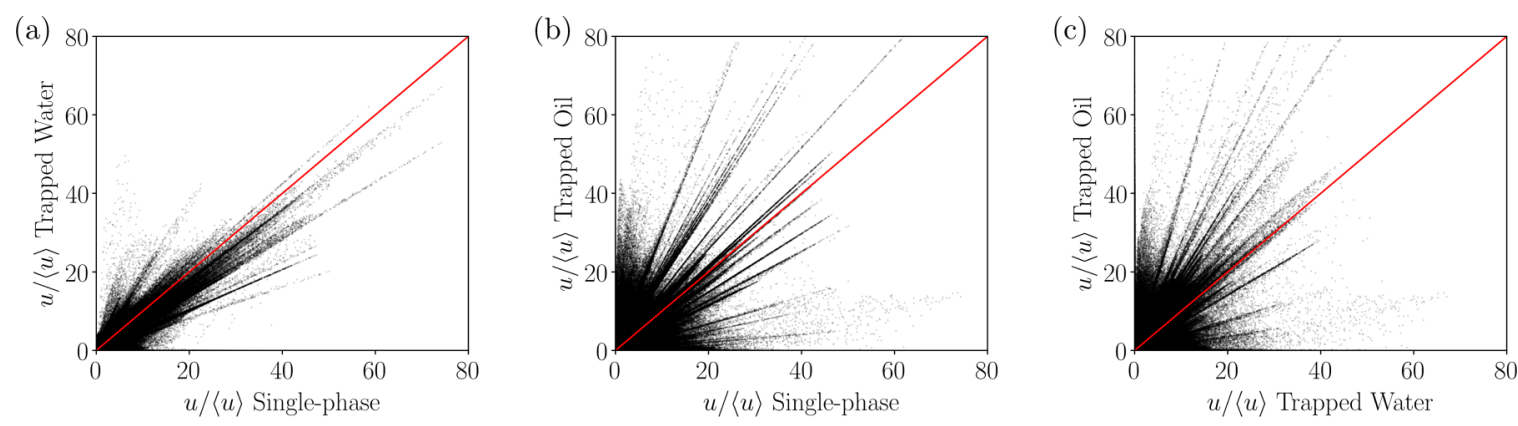

FIG. 6. Scatter plot of scaled velocities juxtaposing (a) trapped water and single-phase conditions, (b) trapped oil and single-phase conditions, or (c) trapped oil and trapped water conditions. Points above and below the parity line are signatures of high-velocity channels present in both cases considered and characterized by increased or decreased velocity magnitudes, respectively. Points close to the horizontal or to the vertical axis are signatures of high-velocity channels that are either disappearing or appearing. 

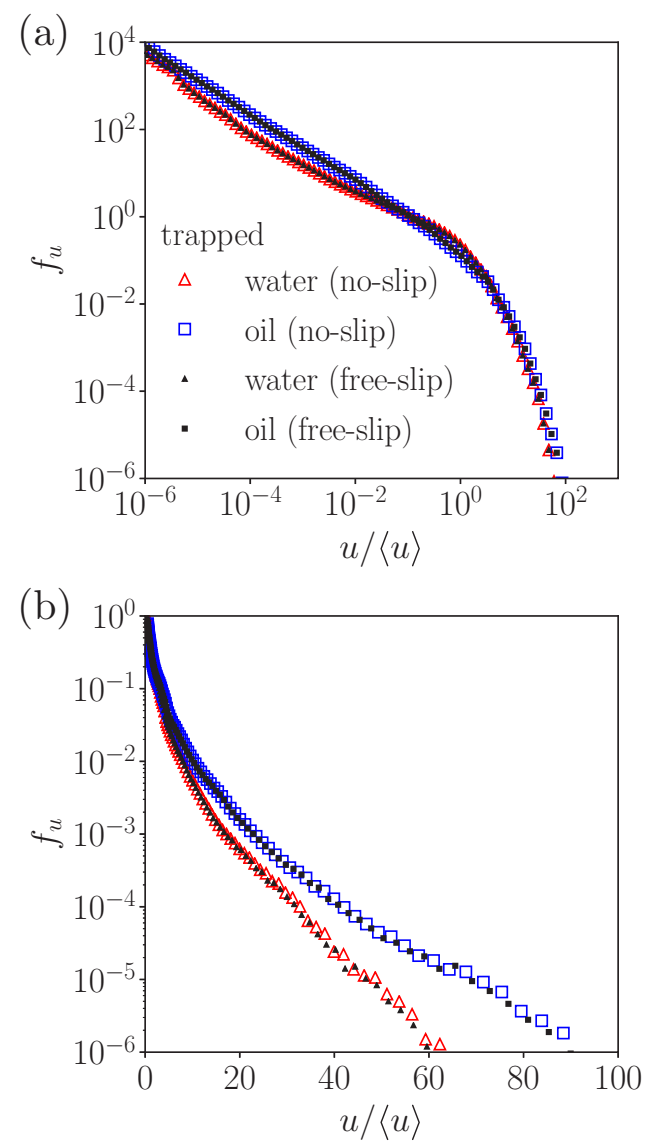

FIG. 7. (a) Probability density functions, $f_{u}$, of scaled velocity magnitude, $u /\langle u\rangle$, for the cases with no-slip conditions (open symbols) and free-slip conditions (plain symbols) implemented at fluid-fluid interfaces (logarithmic scale). (b) Semilogarithmic plot of the same data as in (a) to emphasize high-velocity distribution.

(3) We observe that the presence of a wetting trapped phase yields a distribution of preferential pathways similar to the scenario associated with single-phase conditions. Otherwise, a nonwetting trapped phase has a more pronounced impact on the distribution of preferential pathways.

Our results form the basis to design further numerical and experimental analyses on diverse types of pore spaces to provide a general quantitative assessment of the feedback between velocity distribution features and controlling factors.

\section{ACKNOWLEDGMENTS}

The authors gratefully acknowledge the CINECA award under the ISCRA initiative 2018-2019 (Flo-Tran project) and the Regione Lombardia award under the LISA initiative 2016-2018 (PoreFlow project) for the availability of highperformance computing resources and support. The authors thank P. Lurati for his help during preliminary studies.

\section{APPENDIX A: COMPARISON BETWEEN FLUID-FLUID INTERFACES BOUNDARY CONDITIONS}

Boundary conditions at fluid-fluid interfaces are embedded in the underlying formulation of the two-phase flow model.
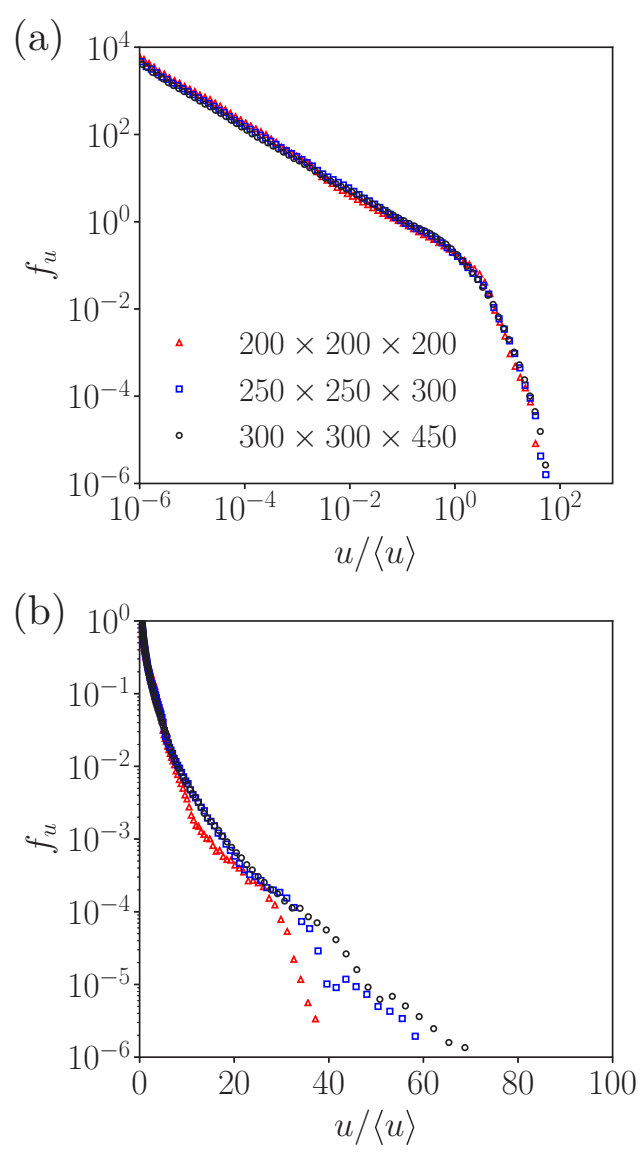

FIG. 8. (a) Probability density functions, $f_{u}$, of scaled velocity magnitude, $u /\langle u\rangle$, obtained under single-phase flow conditions for the diverse sample sizes investigated. (b) Semilogarithmic plot of the same data as in (a) to emphasize high-velocity distribution.

Otherwise, simulation of the flowing phase as a steady-state single-phase system requires setting boundary conditions at fluid-fluid interfaces, the latter being effectively part of the boundary of the solution domain. Highest fidelity would be attained when fluid motion is simulated in both the flowing and trapped phases and coupling of viscous stresses is applied at fluid-fluid interfaces. Practical and efficient implementation of such a coupling for multiple (tens to hundreds of) isolated trapped fluid regions is not straightforward. For instance, when considering the OPENFOAM toolbox, this would imply modifying low-level routines to allow applying a reference pressure to each fluid domain. Disregarding such a feature would yield a diverging numerical solution of the linear system of equations related to the pressure field (continuity equation). As an alternative to this approach, one can resort to a technique which entails considering one of two limit cases, (1) no-slip or (2) free-slip conditions, for which the solution behaves as if the trapped phase had infinite or zero viscosity, respectively. The main advantage of such a simplified approach is that the velocity field in the flowing phase is decoupled from the one in the trapped phase and the solution can be obtained through standard solvers.

Our results are based on the implementation of no-slip conditions at fluid-fluid interfaces. As a comparison, we considered also the implementation of the free-slip conditions. This 
(a)
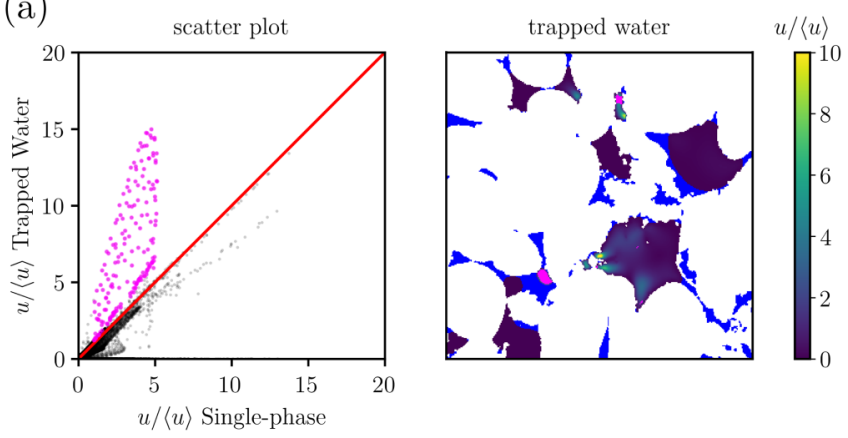

(b)
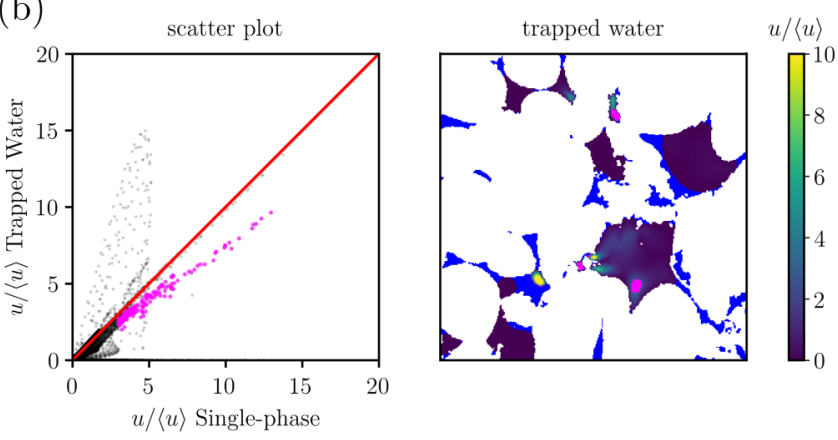

FIG. 9. Selection of points (in magenta) in the scatter plot (a) above and (b) below the parity line (in red), along with the corresponding spatial location, as depicted in magenta in the contour plot of scaled velocity (panels on the right) for the scenario with trapped water. These selections correspond to the points enclosed in the magenta dashed and orange dash-dotted ovals in Fig. 5(a), respectively.

latter case yields generally higher flow rates $(+7 \%$ during drainage and $+1.6 \%$ during imbibition) for the same imposed pressure gradient, as expected. Nevertheless, the probability distributions of scaled velocity magnitude remain unchanged, as illustrated in Fig. 7. This suggests that our conclusions are not affected by the approximation adopted for the treatment of the fluid-fluid interface boundary conditions.

\section{APPENDIX B: INFLUENCE OF THE SAMPLE SIZE}

The rock sample analyzed has a total dimension of $1.2 \times$ $1.2 \times 1.8 \mathrm{~mm}^{3}(300 \times 300 \times 450$ voxels $)$. Smaller subsamples are considered to investigate the influence of the sample size on the results. We focus here on single-phase flow conditions and compare the results in terms of probability density functions of scaled velocity magnitude, $u /\langle u\rangle$. The main characteristics of each subsample analyzed are listed in Table III. (a)
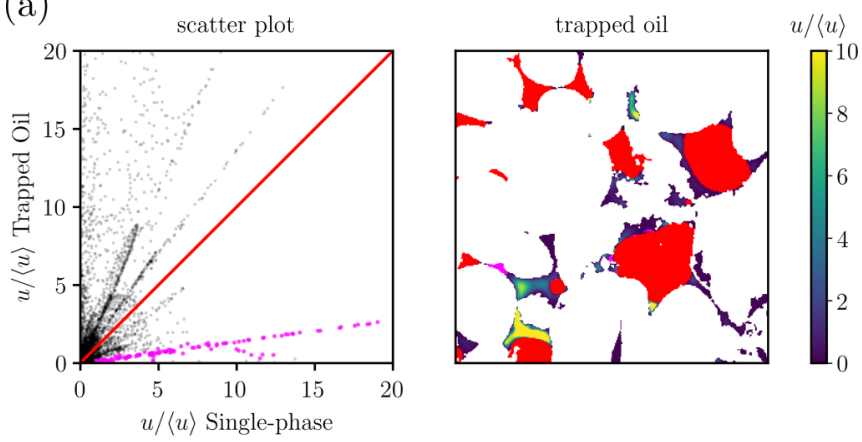

(b)
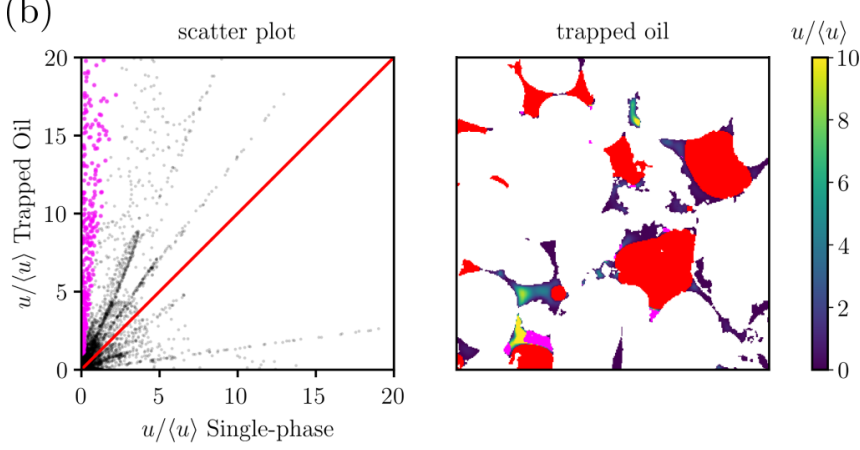

FIG. 10. Selection of points (in magenta) in the scatter plot close to the (a) horizontal and (b) vertical axis, along with the corresponding spatial location, as depicted in magenta in the contour plot of scaled velocity (panels on the right) for the scenario with trapped oil. These selections correspond to the points enclosed in the orange dash-dotted and magenta dashed ovals in Fig. 5(b), respectively.

Considering the low-velocity range [Fig. 8(a)], differences between the cases are minimal, suggesting that the influence of the sample size is negligible in this range. Otherwise, the distribution of high velocities [Fig. 8(b)] associated with the smallest subsample shows some differences as compared to the results documented for the larger samples. These results suggest that relying on the full size sample leads to a stable representation of $f_{u}$.

\section{APPENDIX C: IDENTIFICATION OF MAIN FEATURES IN THE SCATTER PLOTS OF SCALED VELOCITIES}

Scatter plots of scaled velocities corresponding to singlephase flow and fluid flow in the presence of trapped water or oil are characterized by clusters of points forming either linear trends or clouds (see Figs. 5 and 6). Figures 9 and 10 exemplify the correspondence between selections of points in a scatter plot (in magenta) and the corresponding depiction (in

TABLE III. Main characteristics of the subsamples analyzed.

\begin{tabular}{lcccc}
\hline \hline Number of voxels & $\begin{array}{c}\text { Sample size } \\
{\left[\mathrm{mm}^{3}\right]}\end{array}$ & Total porosity & Connected porosity & $\begin{array}{c}\text { Permeability } \\
{\left[\mathrm{m}^{2}\right]}\end{array}$ \\
\hline $200 \times 200 \times 200$ & $0.8 \times 0.8 \times 0.8$ & $14.852 \%$ & $13.977 \%$ & $1.06 \times 10^{-12}$ \\
$250 \times 250 \times 300$ & $1.0 \times 1.0 \times 1.2$ & $12.750 \%$ & $1.485 \%$ & $3.64 \times 10^{-13}$ \\
$300 \times 300 \times 450$ & $1.2 \times 1.2 \times 1.8$ & $12.365 \%$ & $11.465 \%$ & $2.49 \times 10^{-13}$ \\
\hline \hline
\end{tabular}

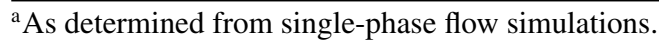


magenta) in the contour plot of scaled velocity. We focus on the four sets of points described in the following.

(1) Figure 9(a) focuses on the selection of points that are above the parity line (in red) and correspond to a high-velocity area experiencing an increase in velocity magnitude with respect to the single-phase scenario, feature A. This selection corresponds to the points enclosed in the magenta dashed ovals in Fig. 5(a).

(2) Figure 9(b) focuses on the selection of points that are below the parity line (in red) and correspond to high-velocity areas experiencing a decrease in velocity magnitude with respect to the single-phase scenario, feature $\mathrm{B}$. This selection corresponds to the points enclosed in the orange dash-dotted ovals in Fig. 5(a).

(3) Figure 10(a) focuses on the selection of points that are close to the horizontal axis and correspond to vanishing high-velocity areas as compared to the single-phase scenario, feature $\mathrm{C}$. This selection corresponds to the points enclosed in the orange dash-dotted ovals in Fig. 5(b).

(4) Figure 10(b) focuses on the selection of points that are close to the vertical axis and correspond to emerging high-velocity areas as compared to the single-phase scenario, feature $\mathrm{D}$. This selection corresponds to the points enclosed in the magenta dashed ovals in Fig. 5(b).

Linear trends observed in the scatter plots may suggest a spatial correlation and might be indicative of points along to the same high-velocity flow path. Otherwise, it should be noted that we also observe high-velocity flow paths that corresponds to clouds of points in the scatter plot. As such, a precise interpretation of such linear trends is still ambiguous, while not strictly related to the main purpose of the study.
[1] F. P. J. de Barros, M. Dentz, J. Koch, and W. Nowak, Geophys. Res. Lett. 39, L08404 (2012).

[2] P. de Anna, T. Le Borgne, M. Dentz, A. M. Tartakovsky, D. Bolster, and P. Davy, Phys. Rev. Lett. 110, 184502 (2013).

[3] Y. Edery, A. Guadagnini, H. Scher, and B. Berkowitz, Water Resour. Res. 50, 1490 (2014).

[4] T. Le Borgne, T. R. Ginn, and M. Dentz, Geophys. Res. Lett. 41, 7898 (2014).

[5] P. K. Kang, T. Le Borgne, M. Dentz, O. Bour, and R. Juanes, Water Resour. Res. 51, 940 (2015).

[6] A. Nissan, I. Dror, and B. Berkowitz, Water Resour. Res. 53, 3760 (2017).

[7] A. Nissan and B. Berkowitz, Phys. Rev. Lett. 120, 054504 (2018).

[8] F. A. Reis, D. Bolster, and V. Voller, Adv. Water Resour. 113, 180 (2018).

[9] Q. Hu and J. S. Wang, Crit. Rev. Environ. Sci. Technol. 33, 275 (2003).

[10] A. Genty and V. Pot, Transp. Porous Media 105, 391 (2014).

[11] J. Jiménez-Martínez, P. de Anna, H. Tabuteau, R. Turuban, T. Le Borgne, and Y. Méheust, Geophys. Res. Lett. 42, 5316 (2015).

[12] A. Honari, M. Zecca, S. J. Vogt, S. Iglauer, B. Bijeljic, M. L. Johns, and E. F. May, Int. J. Greenhouse Gas Control 50, 100 (2016).

[13] R. Yang, E. Lemarchand, and T. Fen-Chong, Transp. Porous Media 111, 347 (2016).

[14] J. Jiménez-Martínez, T. Le Borgne, H. Tabuteau, and Y. Méheust, Water Resour. Res. 53, 1457 (2017).

[15] M. Dentz, M. Icardi, and J. J. Hidalgo, J. Fluid Mech. 841, 851 (2018).

[16] P. de Anna, B. Quaife, G. Biros, and R. Juanes, Phys. Rev. Fluids 2, 124103 (2017).

[17] K. Alim, S. Parsa, D. A. Weitz, and M. P. Brenner, Phys. Rev. Lett. 119, 144501 (2017).

[18] M. Siena, M. Riva, J. D. Hyman, C. L. Winter, and A. Guadagnini, Phys. Rev. E 89, 013018 (2014).

[19] I. Okamoto, S. Hirai, and K. Ogawa, Meas. Sci. Technol. 12, 1465 (2001).
[20] S. S. Datta, H. Chiang, T. S. Ramakrishnan, and D. A. Weitz, Phys. Rev. Lett. 111, 064501 (2013).

[21] OpenCFD Limited, OpenFOAM The Open Source CFD Toolbox, user guide version v1612, https://www.openfoam.com/ (2016).

[22] H. Rusche, Computational fluid dynamics of dispersed twophase flows at high phase fractions, Ph.D. thesis, Department of Mechanical Engineering, Imperial College of Science, Technology \& Medicine, London, 2002.

[23] J. Brackbill, D. Kothe, and C. Zemach, J. Comput. Phys. 100, 335 (1992).

[24] M. M. Francois, S. J. Cummins, E. D. Dendy, D. B. Kothe, J. M. Sicilian, and M. W. Williams, J. Comput. Phys. 213, 141 (2006).

[25] S. O. Unverdi and G. Tryggvason, J. Comput. Phys. 100, 25 (1992).

[26] J. T. Gostick, Phys. Rev. E 96, 023307 (2017).

[27] G. R. Guédon, J. D. Hyman, F. Inzoli, M. Riva, and A. Guadagnini, Phys. Fluids 29, 123104 (2017).

[28] T. Ramstad, N. Idowu, C. Nardi, and P.-E. Øren, Transp. Porous Media 94, 487 (2012).

[29] P. Meakin and A. M. Tartakovsky, Rev. Geophys. 47, RG3002 (2009).

[30] R. Issa, J. Comput. Phys. 62, 40 (1986).

[31] Y. Saad, Iterative Methods for Sparse Linear Systems, 2nd ed. (Society for Industrial and Applied Mathematics, Philadelphia, 2003).

[32] F. Denner and B. G. van Wachem, J. Comput. Phys. 285, 24 (2015).

[33] S. Patankar and D. Spalding, Int. J. Heat Mass Transf. 15, 1787 (1972).

[34] L. Moghadasi, A. Guadagnini, F. Inzoli, M. Bartosek, and D. Renna, J. Pet. Sci. Eng. 145, 453 (2016).

[35] M. J. Blunt, Multiphase Flow in Permeable Media (Cambridge University Press, Cambridge, 2017).

[36] CINECA, https://www.cineca.it/en (2018).

[37] M. Siena, J. D. Hyman, M. Riva, A. Guadagnini, C. L. Winter, P. K. Smolarkiewicz, P. Gouze, S. Sadhukhan, F. Inzoli, G. Guédon, and E. Colombo, Comput. Geosci. 19, 423 (2015). 